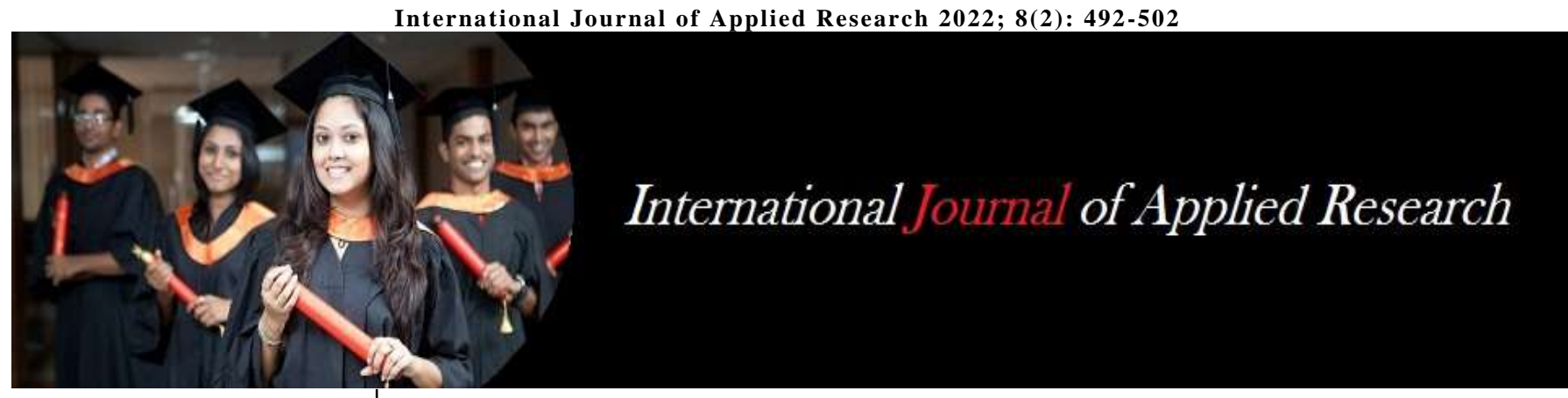

ISSN Print: 2394-7500 ISSN Online: 2394-5869 Impact Factor: 8.4 IJAR 2022; 8(2): 492-502 www.allresearchjournal.com Received: 12-12-2021 Accepted: 17-01-2022

Dr. Tejaswini Vasave MPT (Musculoskeletal) PES Modern College of Physiotherapy, Pune, Maharashtra, India

Dr. Sanket Nagrale Associate Professor, Musculoskeletal Department, PES Modern College of Physiotherapy, Pune, Maharashtra, India

Corresponding Author: Dr. Tejaswini Vasave MPT (Musculoskeletal) PES Modern College of Physiotherapy, Pune, Maharashtra, India

\section{Effect of lumbar high velocity low amplitude (HVLA) thrust on speed and agility in elite athletes: A randomised controlled trial}

\section{Dr. Tejaswini Vasave and Dr. Sanket Nagrale}

\author{
DOI: $\underline{\text { https://doi.org/10.22271/allresearch.2022.v8.i2g.9487 }}$
}

\begin{abstract}
Elite athletes constantly seek methods to enhance performance ${ }^{[1]}$. Pre-competition manipulation is a prevalent but underappreciated technique.

${ }^{[1]}$ Depending on the assessment, HVLA thrust of the spinal or extremities joint is frequently used ${ }^{[1]}$. A manipulation is a single high-velocity thrust administered to a joint, or a sequence of joints, and the soft tissues around them ${ }^{[2]}$. Usually, the goal is to alleviate discomfort ${ }^{[2]}$. These treatments are only used on a patient who is completely relaxed ${ }^{[2]}$. A manipulative push technique extends the joint's typical physiological range for a brief period of time ${ }^{[2]}$. Accurate localisation of the joint position before to the procedure, followed by a carefully delivered thrust, will result in a movement that is so quick that the patient will barely notice it has started ${ }^{[2]}$. HVLA thrust may boost performance since it facilitates motor neuron pool excitability for 20-60s, according to basic physiological studies. When conducted on the joints of the lumbar spine, it results in a considerable increase in surface electromyographically measured erector spinae isometric maximal voluntary contraction muscle output and is helpful in providing short-term pain relief ${ }^{[1]}$. HVLA thrust, a type of spinal manipulation, has been demonstrated to activate the brain in studies. Mechanoreceptors and proprioceptors from structures in and around the manipulated joint ${ }^{[40]}$. The altered afferent input arising from the stimulation of these receptors is thought to cause changes in motor neuron excitability, which then results in local or regional muscular changes around the manipulation site ${ }^{[40]}$. Manipulative treatment has been provided as standard of care to athletes at Olympic Games and other international multisport games ${ }^{[41]}$. Pre competition manipulation may help enhance musculoskeletal function by warming up soft tissues to optimize joint function ${ }^{[41]}$. Many articles have shown significant immediate effects of HVLA, however not many studies have focused on long term effects. Shrier I et al. did a pilot study that showed pre event manipulation improved the sprint time in athletes ${ }^{[1]}$. Therefore, this study will be focusing on effects of lumbar HVLA thrust on speed and agility in elite athletes.

Objectives:
\end{abstract}

1. To check the effect of lumbar HVLA on speed in elite athletes after 2 weeks.

2. To check the effect of lumbar HVLA on agility in elite athletes after 2 weeks.

Methodology: With the permission of ethical committee the study was started. Consent was taken from the athletes participating in the study. 42 players were selected for the study based on the inclusion criteria. They were randomly divided into 2 groups Group A (Interventional) $(\mathrm{N}=22)$ and Group B (Control) $(\mathrm{N}=20)$. Single blinding method was used in this study in which the athletes were kept ignorant of either of the group they are assigned to but the therapist was in possession of this knowledge Group A was given HVLA along with conventional. Group B was given conventional training. Pre intervention speed and agility were recorded in both groups. Post intervention (post 2 weeks) speed and agility was done again in both the groups. For interventional group and control group the training protocol was composed of $10 \mathrm{mins}$ warm up exercises with stretching, 40 minutes of exercises with adequate breaks in between, 10mins of cool down exercise.

Data was analysed using Wilcoxon rank t test in Group A within the group and Paired t test in group B within the group. And unpaired t test was used to analyse the data of speed between group A \& B and Mann Whitney test for agility between group A \& B.

Result: HVLA thrust showed non-significant improvement (p value- 0.12) for speed in elite athletes when compared to conventional training. HVLA thrust showed not quite significant improvement ( $p$ value-0.07) for agility in elite athletes when compared to conventional training.

Conclusion: Interventional group showed clinically significant improvement within the group. But on comparing with the control group the results were not significant. Further studies are needed with larger sample size to find the effects of HVLA thrust in performance of elite athletes.

Keywords: HVLA, elite athletes, thrust, speed, agility

\section{Introduction}

Elite athletes constantly seek methods to enhance performance ${ }^{[1]}$. A varsity player (individual or team), a professional player, or a national or international level player who is presently or has previously competed ${ }^{[5]}$. The increased competitiveness and demand in the sports field has resulted in a greater search for alternatives that can help athletes improve 
their performance, particularly those who do not use medications or undergo surgery. Structure and functionally healthy tissue, as well as a neuromuscular coordination network, are essential to compete at high levels. These factors play a direct role in a successful sports career, where endurance, strength, flexibility, and proprioception are essential ${ }^{[6]}$. Manual treatments are one of the many instruments utilised in the sports world, and they include a wide range of procedures that can assist athletes improve their performance ${ }^{[6]}$. Manipulation is a passive method in which the therapist administers a precise manual impulse, or thrust, to a joint at or near the end of its passive (or physiological) range of motion. This is frequently accompanied by a loud 'crack.' The fact that spinal manipulation techniques produce a pop or cracking sound within synovial joints is a typical feature. Although the mechanism of this audible discharge is unknown, it is usually assumed to be cavitation of a spinal facet joint.

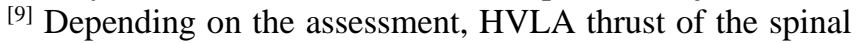
or extremities joint is frequently used ${ }^{[1]}$. A manipulation is a single high-velocity thrust administered to a joint, or a sequence of joints, and the soft tissues around them ${ }^{[2]}$. Usually, the goal is to alleviate discomfort ${ }^{[2]}$. In soccer, athletics, and golf, high-velocity low-amplitude manipulation (thrust) has been utilised to increase performance.

A quick and short pulse is administered to the end of the synovial joints' range of passive motion in this kind of handling ${ }^{[6]}$. These procedures are only used on a patient who is completely relaxed [2]. A manipulative push technique extends the joint's typical physiological range for a brief period of time ${ }^{[2]}$. Accurate localisation of the joint position before to the method, followed by a deftly executed thrust, results in a movement that is practically complete before the patient realises it has started ${ }^{[2]}$. HVLA thrust may boost performance since it facilitates motor neuron pool excitability for 20-60s, according to basic physiological studies ${ }^{[1]}$. It produces a significant increase in surface electromyographically measured erector spinae isometric maximum voluntary contraction muscle output when performed on the joints of the lumbar spine, and is effective in producing short term pain relief ${ }^{[1]}$. Studies have shown that spinal manipulation in the form of HVLA thrust activates mechanoreceptors and proprioceptors from structures in and around the manipulated joint. [3] Mechanoreceptors detects changes in balance, touch, proprioception and pain (experienced in burning sensation). ${ }^{[1]}$ The altered afferent input arising from the stimulation of these receptors is thought to cause changes in motor neuron excitability, which then results in local or regional muscular changes around the manipulation site. ${ }^{[3]}$ The HVLA thrust is equivalent to rapidly applying a mechanical strain to the trunk and, as such, appears to be the critical factor in reflex activation of paraspinal muscles. Reflex activation of paraspinal muscles in response to mechanical perturbations triggers a prolonged reduction of motoneuron activity ${ }^{[7]}$.

Athletes at the Olympic Games and other international multisport events have received manipulative treatment as standard of care ${ }^{[4]}$. Warming up soft tissues before a competition can aid improve musculoskeletal function and joint function ${ }^{[4]}$. In a study by Sandell, Palmgren, and Björndahl, the experimental group's running speed, which was calculated based on the time it took to cross a given distance, was higher ${ }^{[8]}$ In a study done by Shrier, MacDonald and Uchacz concluded that the pre event manipulation was beneficial on the explosive power (tested with means of vertical jump) and on running velocity or agility [1]. Agility is defined as a rapid whole-body movement with change of velocity or direction in response to a stimulus ${ }^{[10]}$. Many articles have shown significant immediate effects of HVLA, however not many studies have focused on medium/long term effects ${ }^{[4]}$. Therefore, this study will be focusing on effects of lumbar HVLA thrust on speed and agility in elite athletes.

\section{Aim \& objectives}

AIM- To find the effect of lumbar HVLA thrust on speed \& agility in elite athletes

\section{Objectives}

1. To check the effect of lumbar HVLA thrust on speed in elite athletes after 2 weeks.

2. To check the effect of lumbar HVLA thrust on agility in elite athletes after 2 weeks

\section{Hypothesis}

Null Hypothesis -

There will be no significant effect of lumbar HVLA thrust on speed and agility in elite athletes after 2 weeks

Alternate Hypothesis (H1) -

There will be a significant effect of lumbar HVLA thrust on speed in elite athletes after 2 weeks.

Alternate Hypothesis (H2) -

There will be a significant effect of lumbar HVLA thrust on agility in elite athletes after 2 weeks.

\section{Materials \& Methods -}

Research design: Randomized control trial, single blinded. Source of data: The data was collected from the sport clubs centres in and around the metropolitan city. The study included 42 athletes who were elite athletes (national level) of age group between 18 to 35 years, who satisfied the inclusion and exclusion criteria's.

Type of data: The data was primary which was collected by the principle investigator.

Duration of study: The total duration of the study was one year. Intervention period was for 60 minutes per session in a day, 2 days per week for 2 weeks, which was given to both the group.

Sample size: Data was obtained from previous study to calculate the sample size for the current study. Sample Size was calculated using GPower 3.1 version.

T tests - Means: Difference between two independent means (two groups)

Analysis: A priori: Compute required sample size

Input: Tail(s) $=$ Two Effect size $\mathrm{d}=0.8 \alpha$ err prob $=0.05$ Power $(1-\beta$ err prob $)=0.8$ Allocation ratio N2/N1 $=1$

\section{Output}

Noncentrality parameter $\delta=2.8844410$

Critical $\mathrm{t}=2.0085591$

Df $=50$ 
Sample size group $1=26$

Sample size group $2=26$

Total sample size $=52$

Actual power $=0.8074866$

Note: Due to Covid-19 Pandemic Situation in India all the samples were not collected as calculated earlier.

The final samples were 22 samples in Group 1 \& 20 samples in Group 2

Sampling method: Purposive Random Sampling Computer generated randomization was done. Randomization sequence was concealed in a sequentially numbered envelop. The subject was then asked to pick an envelope and were allotted to the group based on the number written on the paper inside the envelope. In group A \& group B.

Selection Criteria: Inclusion Criteria:

Males ${ }^{[19]}$

Age Group 18-35 [19]

Elite Athletes who have completed 5 years of training in a club.
$\mathrm{T}$ test score ranging from average to good (9.5-11.5 seconds) ${ }^{[20]}$

Exclusion Criteria:

Recent Musculoskeletal injury likely to affect the performance ${ }^{[1]}$

Undiagnosed pain ${ }^{[2]}$

Any tingling sensation ${ }^{[2]}$

Generalized hypermobility ${ }^{[2]}$

Severe degenerative changes in the affected joint ${ }^{[2]}$

Vascular abnormalities, e.g. vertebral artery involvement ${ }^{[2]}$

\section{Outcome measures}

1) Speed $100 \mathrm{~m}$ sprint performance was checked using a stop watch and the time was documented.

Speed $=$ distance $/$ time

2) Agility T-Test

Reliability $0.98^{[20]}$

The T-test is a popular test of change of direction speed (Pauole et al. 2000; Semenick 1990).

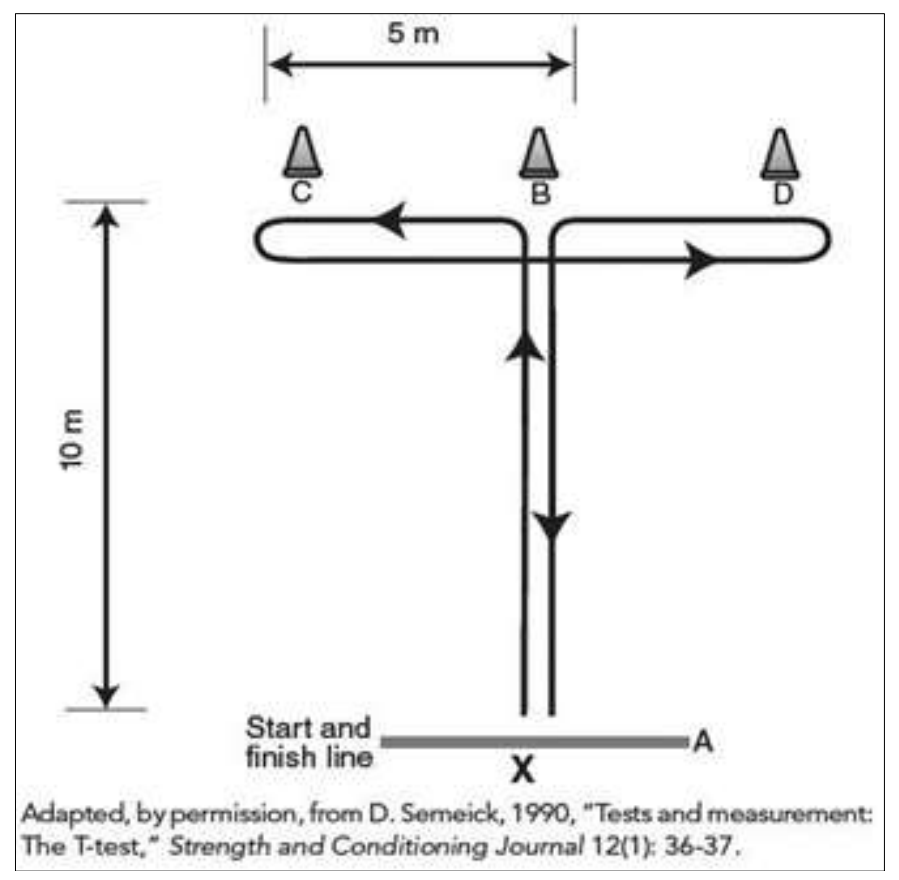

Fig 1: Schematic illustration of the T-test.

The T-test was administered using the protocol outlined by Semenick ${ }^{[22]}$ with minor modifications. Specifically, bells were placed at the base of 3 cones (B, C, and D) in an attempt to monitor consistency and accuracy of test execution. Subjects began with both feet behind the starting point A. At their own discretion, each subject sprinted forward $10 \mathrm{~m}$ to point $\mathrm{B}$ and rang a bell at the base of a cone with the right hand. They then shuffled to the left $5 \mathrm{~m}$ and touched a bell at the base of a cone (C) with the left hand.

Subjects then shuffled to the right $5 \mathrm{~m}$ m and touched a bell at the base of a cone (D) with the right hand. They then shuffled to the left $5 \mathrm{~m}$ back to point $\mathrm{B}$ and touched a bell with the left hand. Subjects then ran backward, passing the finishing line at point $\mathrm{A}$.

Three test trials were performed and were recorded with a stop watch. The test was repeated if a subject failed to ring the bell at the base of each cone, crossed his feet when shuffling, and/or did not face forward at all times. The fastest trial was used for statistical analyses.

\section{Materials used}

- Pen

- Measuring tape

- Marker

- Case record sheet

- Cones

- Stop watch

- Mat

\section{Procedure}

With the permission of ethical committee the study was started. Consent was taken from the athletes participating in the study. 42 players were selected for the study based on 
the inclusion criteria. They were randomly divided into 2 groups Group A (Interventional) and Group B (Control). Single blinding method was used in this study in which the athletes were kept ignorant of either of the group they are assigned to but the therapist was in possession of this knowledge Group A was given HVLA along with conventional. Group B was given conventional training. Pre intervention speed and agility were recorded in both groups. Post intervention (post 2 weeks) speed and agility was done again in both the groups. For interventional group and control group the training protocol was composed of $10 \mathrm{mins}$ warm up exercises with stretching, 40 minutes of exercises with adequate breaks in between, 10mins of cool down exercise.

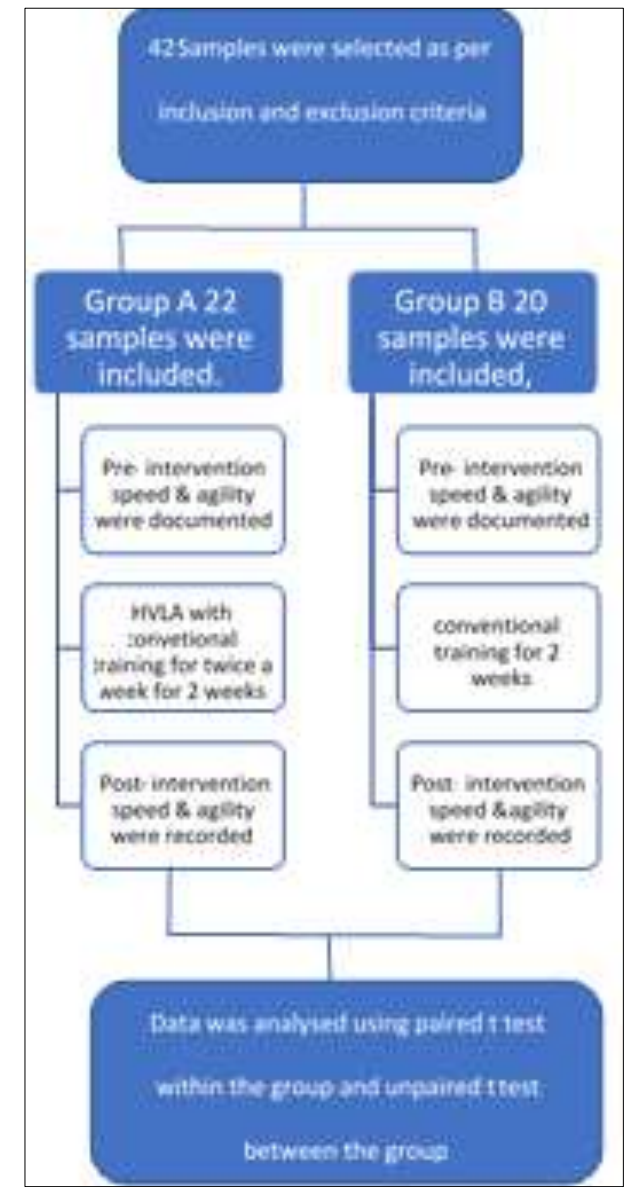

\section{Conventional Training Protocol}

1) Warm up Exercises

Shoulder circles, Multidirectional Lunges, Slow run.

\section{Stretching}

Static stretch for 30 seconds bilaterally - Hamstring Stretch, Adductor Stretch, Quadratus Lumborum Stretch, Quadriceps, Gastro-Soleus.

Table 1: Exercises

\begin{tabular}{|c|c|c|}
\hline Training Week & Exercises & Sets*Repetitions \\
\hline \multirow{8}{*}{ Week 1} & 1) Jump squats & \multirow{8}{*}{2 sets $* 10$ reps each } \\
\hline & 2) Kick Drills & \\
\hline & 3) High Knees & \\
\hline & 4) Lunges & \\
\hline & 5) Bridges & \\
\hline & 6) Sit-ups & \\
\hline & 7) Push ups & \\
\hline & 8) Triceps-dips & \\
\hline \multirow{8}{*}{ Week 2} & 1) Jump squats & \multirow{8}{*}{2 sets $* 15$ reps each } \\
\hline & 2) Kick Drills & \\
\hline & 3) High Knees & \\
\hline & 4) Lunges & \\
\hline & 5) Bridges & \\
\hline & 6) Sit-ups & \\
\hline & 7) Push ups & \\
\hline & 8) Triceps-dips & \\
\hline
\end{tabular}




\section{Exercises}
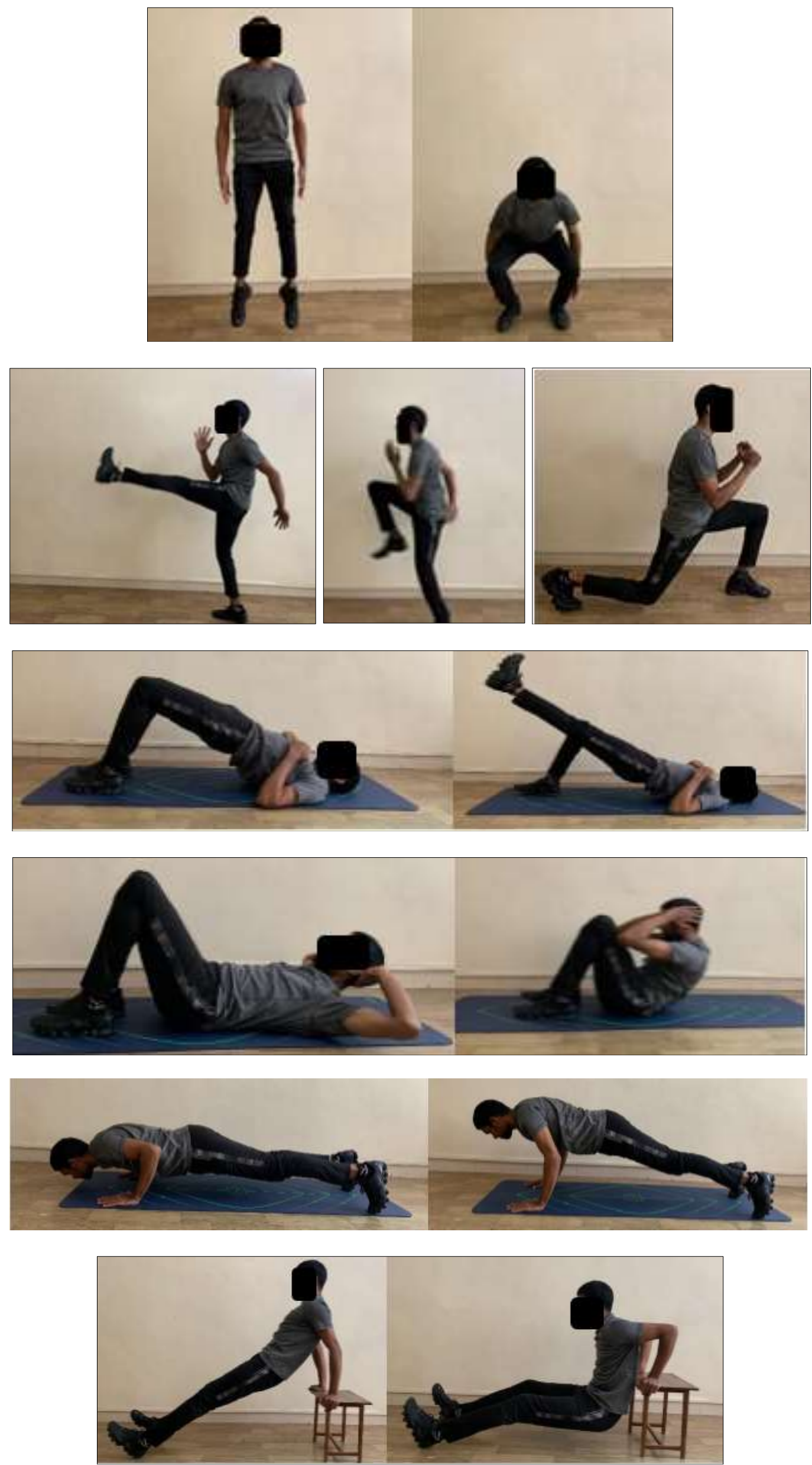


\section{Lumbar HVLA thrust}

Thrust was given twice in a week for 2 weeks.

The patient is placed in the left side-lying position on the plinth. The right hip is flexed until motion is palpated at the interspinous space at L4-5. The torso of the patient is then rotated right until motion is again felt at the same space. With the patient in proper position, the therapist provides a HVLA rotational thrust of the pelvis anteriorly and inferiorly. A 'pop' or 'cracking sound' should be heard. Same is repeated on the other side.

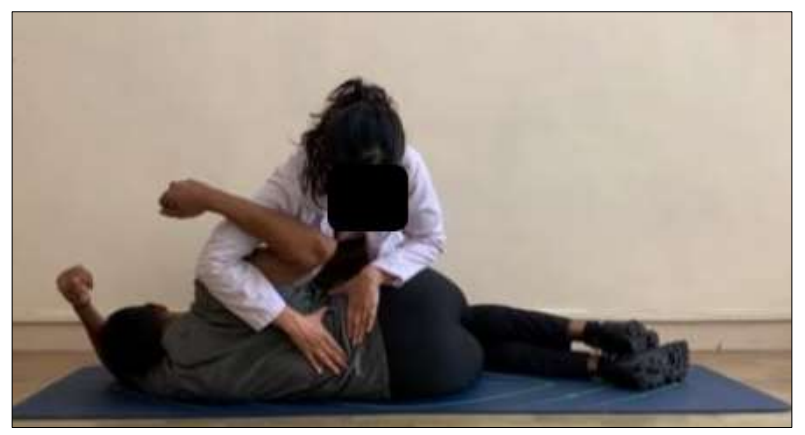

\section{Data analysis and results}

The data collected by the primary investigator after 2 weeks of exercises and HVLA thrust in elite athletes was entered into excel spread sheet, tabulated and subjected to statistical analysis.

The Data was analysed with the help of Instat Graphpad version 3.1

Various statistical measures such as mean, standard deviation (SD) and test of significance were utilized to analyse the data. The results were concluded to be statistically significant if, $\mathrm{p}<0.05$

\section{Results}

Total 22 athletes in group A and 20 athletes in group B Further samples couldn't be collected due to Covid-19 Pandemic situation, Results are based on the samples collected.

Table 2: Age in Group A \& Group B

\begin{tabular}{|c|c|c|c|c|c|c|}
\hline \multirow{2}{*}{ Parameter } & \multicolumn{2}{|c|}{ Group A } & \multicolumn{2}{c|}{ Group B } & \multirow{2}{*}{ P value } & \multirow{2}{*}{ Result } \\
\cline { 2 - 5 } & Mean & SD & Mean & SD & & \multirow{2}{*}{ Not Significant } \\
\hline Age(years) & 22.04 & 2.05 & 21.5 & 2.16 & 0.40 & \\
\hline
\end{tabular}

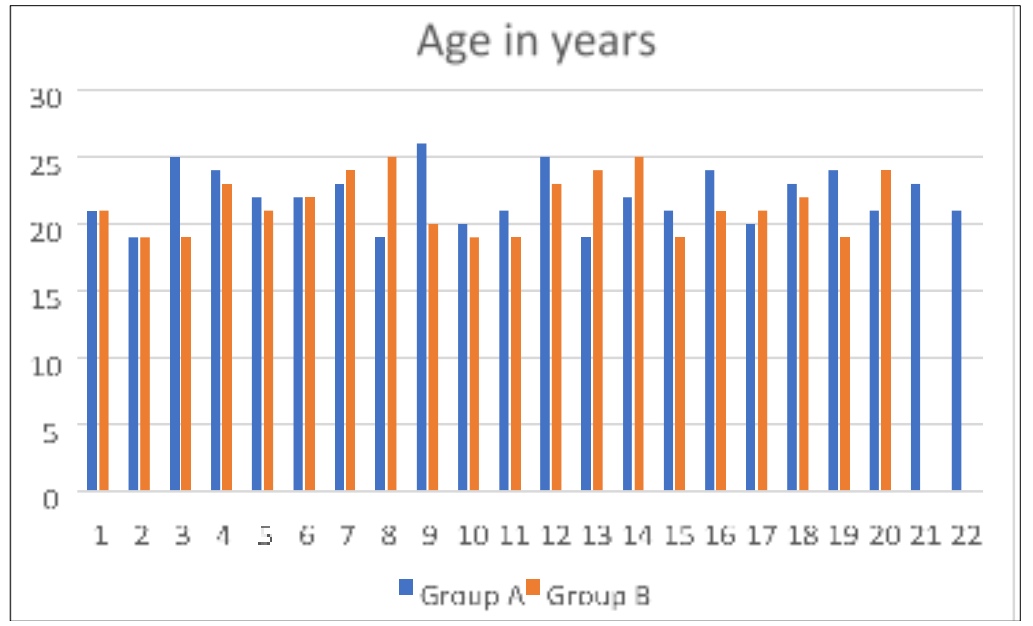

Table 3: Height in Group A \& B

\begin{tabular}{|c|c|c|c|c|c|c|}
\hline \multirow{2}{*}{ Parameter } & Group A & & Group B & & \multirow{2}{*}{ P value } & \multirow{2}{*}{ Result } \\
\cline { 2 - 5 } & Mean & SD & Mean & SD & & Not Significant \\
\hline Height $(\mathrm{cm})$ & 171.18 & 2.32 & 171.05 & 2.11 & 0.84 & N \\
\hline
\end{tabular}




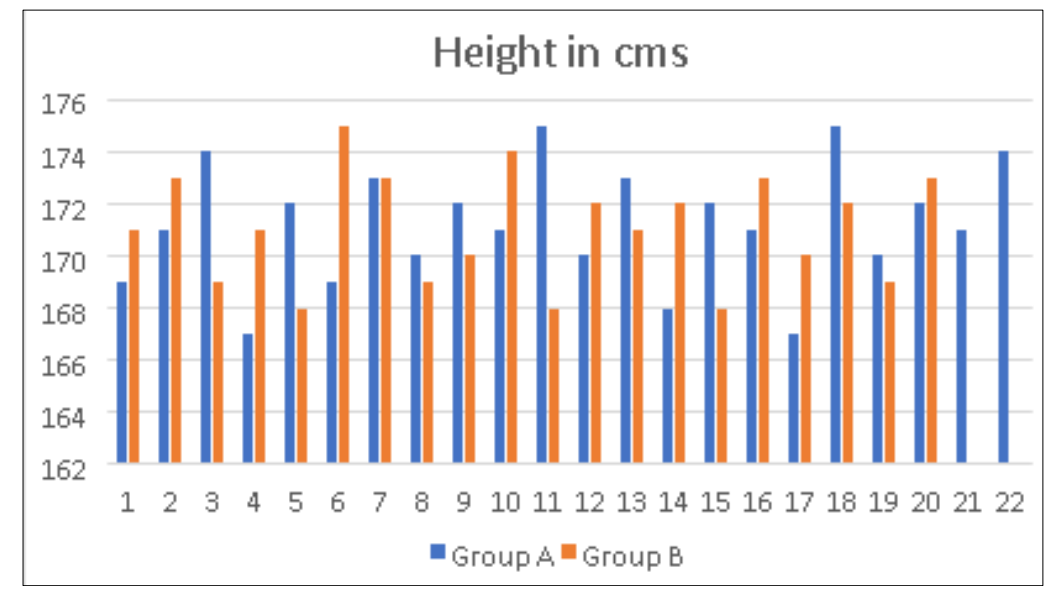

Table 4: Comparison of Pre \& Post of Speed in elite athletes in Group A using Wilcoxon Match Paired test

\begin{tabular}{|c|c|c|c|c|c|c|c|c|}
\hline \multirow{2}{*}{ Parameters } & \multicolumn{2}{|c|}{ Pre test } & \multicolumn{2}{c|}{ Post test } & \multicolumn{2}{c|}{ 95\% Confidence interval } & \multirow{2}{*}{ P value } & \multirow{2}{*}{ Result } \\
\cline { 2 - 8 } & Mean & SD & Mean & SD & Lower & Upper & & \\
\hline Speed $(\mathrm{m} / \mathrm{s})$ & 9.21 & 0.30 & 9.32 & 0.22 & 0.19 & 0.02 & 0.0053 & Significant \\
\hline
\end{tabular}

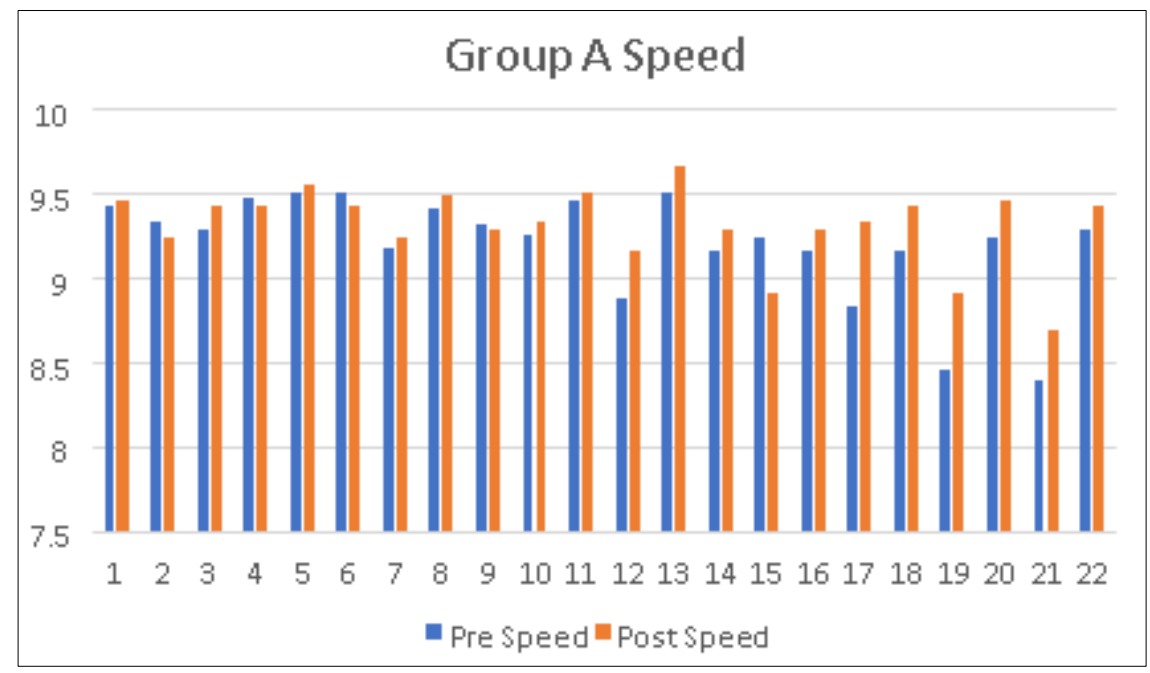

Table 5: Comparison of Pre \& Post of Speed in elite athletes in Group B using Paired t test

\begin{tabular}{|c|c|c|c|c|c|c|c|c|}
\hline \multirow{2}{*}{ Parameters } & \multicolumn{2}{|c|}{ Pre test } & \multicolumn{2}{c|}{ Post test } & \multicolumn{2}{c|}{ 95\% Confidence Interval } & \multirow{2}{*}{ P value } & \multirow{2}{*}{ Result } \\
\cline { 2 - 9 } & Mean & SD & Mean & SD & Lower & Upper & & Not Significant \\
\hline Speed $(\mathrm{m} / \mathrm{s})$ & 9.26 & 0.20 & 9.29 & 0.17 & 0.10 & 0.05 & 0.48 & N \\
\hline
\end{tabular}

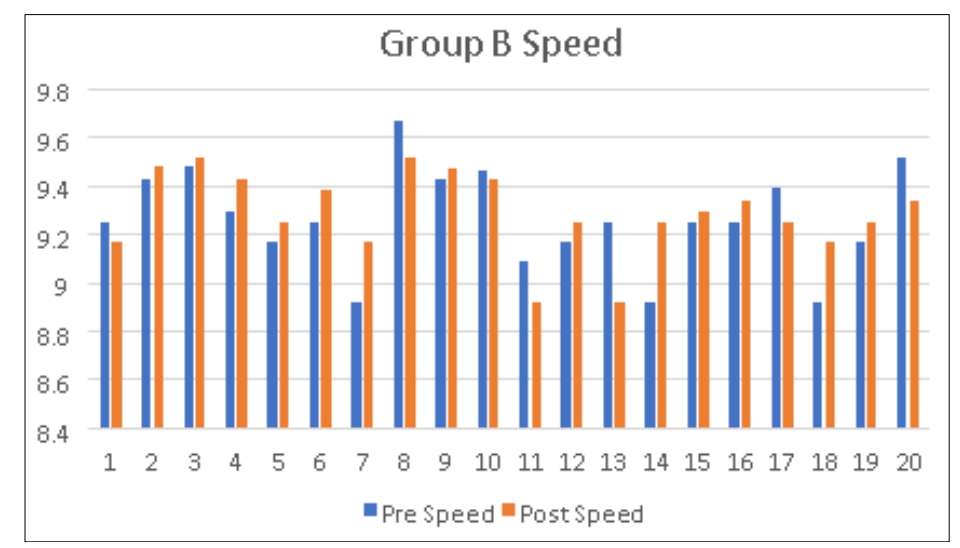

Table 6: Comparison of Pre \& Post of Agility in elite athletes in Group A using Wilcoxon Matched Paired test-

\begin{tabular}{|c|c|c|c|c|c|c|c|c|}
\hline \multirow{2}{*}{ Parameters } & \multicolumn{2}{|c|}{ Pre test } & \multicolumn{2}{c|}{ Post test } & \multicolumn{2}{c|}{ 95\% Confidence interval } & \multirow{2}{*}{ P value } & \multirow{2}{*}{ Result } \\
\cline { 2 - 9 } & Mean & SD & Mean & SD & Lower & Upper & \multirow{2}{*}{ Significant } \\
\hline Agility (sec) & 9.66 & 0.35 & 9.82 & 0.38 & 0.006 & 0.29 & 0.035 & (29 \\
\hline
\end{tabular}




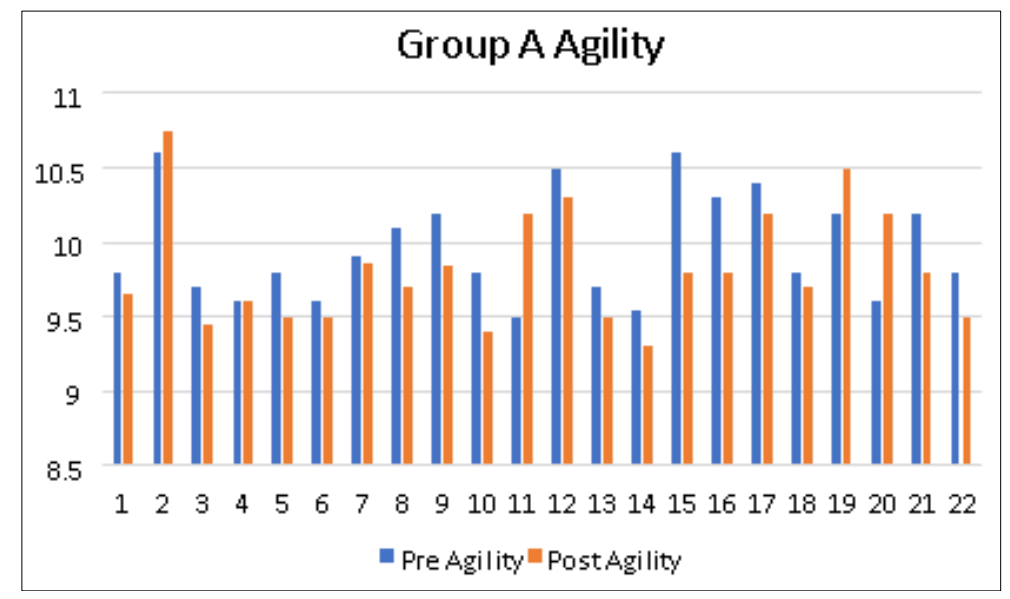

Table 7: Comparison of Pre \& Post of Agility in elite athletes in Group B using Paired t test

\begin{tabular}{|l|c|c|c|c|c|c|c|c|}
\hline \multirow{2}{*}{ Parameters } & \multicolumn{2}{|c|}{ Pre test } & \multicolumn{2}{c|}{ Post test } & \multicolumn{2}{c|}{ 95\% confidence interval } & \multirow{2}{*}{ P value } & \multirow{2}{*}{ Result } \\
\cline { 2 - 9 } & Mean & SD & Mean & SD & Lower & Upper & & Not Significant \\
\hline Agility (sec) & 9.87 & 0.21 & 9.82 & 0.24 & 0.03 & 0.14 & 0.20 & \\
\hline
\end{tabular}

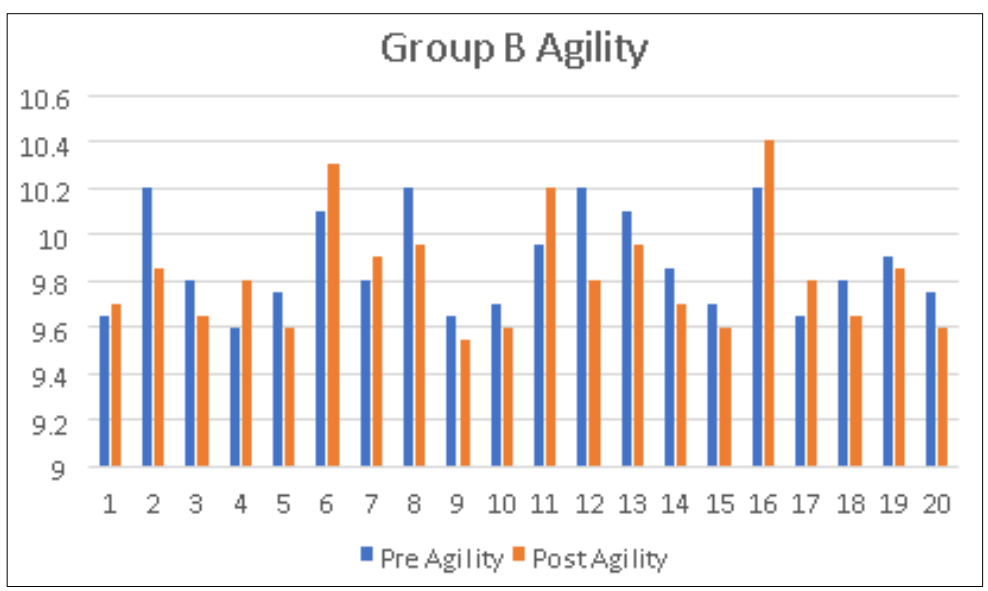

\section{Intragroup analysis}

The pre \& post intervention data for Group A for speed and agility was analysed using Wilcoxon Matched Paired test. Group A data did not pass the normality. Both Speed and Agility were statistically significant.
The pre \& post intervention data for Group B for speed and agility was analysed using Paired t test. Group B data passed the normality. Both Speed and agility were statistically no significant.

Table 8: Comparison of speed between Group A \& B using unpaired t-test

\begin{tabular}{|c|c|c|c|c|c|c|c|c|c|c|c|c|}
\hline \multirow{2}{*}{ Parameters } & \multicolumn{2}{|c|}{ Group A } & & & & \multicolumn{2}{|c|}{ Group B } & & & & \multirow{2}{*}{$P$ value } & \multirow{2}{*}{ Result } \\
\hline & Mean & SD & SEM & \multicolumn{2}{|c|}{$95 \%$ CI } & Mean & SD & SEM & & CI & & \\
\hline \multirow{2}{*}{ Speed $(\mathrm{m} / \mathrm{s})$} & \multirow{2}{*}{0.11} & \multirow{2}{*}{0.18} & \multirow{2}{*}{0.03} & Lower & Upper & \multirow{2}{*}{0.02} & \multirow{2}{*}{0.16} & \multirow{2}{*}{0.03} & Lower & Upper & \multirow{2}{*}{0.124} & \multirow{2}{*}{ Not Significant } \\
\hline & & & & 0.02 & 0.19 & & & & 0.05 & 0.10 & & \\
\hline
\end{tabular}

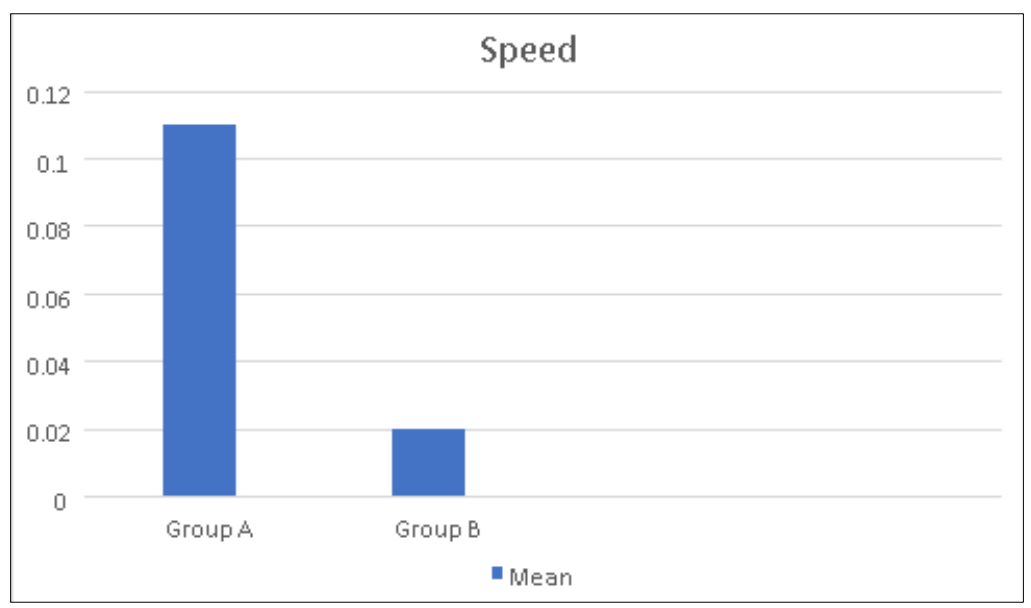


Table 9: Comparison of agility between Group A \& B using Mann Whitney test

\begin{tabular}{|c|c|c|c|c|c|c|c|c|c|c|c|c|}
\hline \multirow{2}{*}{ Parameters } & \multicolumn{2}{|c|}{ Group A } & & & & \multicolumn{2}{|c|}{ Group B } & & & & \multirow{2}{*}{$P$ value } & \multirow{2}{*}{ Result } \\
\hline & Mean & SD & SEM & \multicolumn{2}{|c|}{$95 \% \mathrm{CI}$} & Mean & SD & SEM & $95^{\circ}$ & CI & & \\
\hline \multirow{2}{*}{ Agility (sec) } & 014 & 034 & 007 & Lower & Upper & \multirow{2}{*}{0.05} & \multirow{2}{*}{0.18} & \multirow{2}{*}{0.04} & Lower & Upper & \multirow{2}{*}{0.07} & \multirow{2}{*}{ Not quite significan } \\
\hline & 0.14 & 0.34 & 0.07 & 0.29 & 0.006 & & & & 0.14 & 0.03 & & \\
\hline
\end{tabular}

\section{Intergroup analysis}

The difference of speed for Group A (interventional group) \& group B (control group) was analysed using Unpaired $t$ test. The mean score difference was non-significant ( $p$ value- 0.12)

The difference of agility for Group A (interventional group) \& group B (control group) was analysed using MannWhitney test. The mean score difference was not quite significant (p value- 0.07 )

\section{Discussion}

The result of this study implied that HVLA showed clinical as well as statistically significant improvement within the group. Although when compared to the control group the results were non-significant. To our knowledge this is the first study that checked the effect of HVLA on 100m speed and agility. Two articles analysed the effects of thrust on the running speed, with a total of 34 volunteers enrolled ${ }^{[1,8]}$. Our study focused on long term effects of HVLA on running speed on athletes to check whether the effect of HVLA is long lasting. The thrust was given twice in a week for two weeks along the conventional training the athlete is undergoing. As reviewed by Pasquier et al. ${ }^{[27]}$ showed that if the treatment is repeated over a period of time compared to no treatment or light massage, SM therapy had durable benefits for neuromusculoskeletal disorders-related pain and/or disability. When frequency effects are considered for patients with back pain or headache, trends in improvement were observed and indicated that increasing the number of $\mathrm{SM}$ visits in a short period of time (few weeks) decreased pain and improve disability as well as reducing the number of days with headaches episodes. As accordance to this review we gave HVLA thrust twice in a week for two weeks along with the conventional training to the elite athletes. The duration was kept 2 weeks as the athletes had many university and state level competitions going on while we conducted our study in our city. Shrier et al. checked on 20 to $60 \mathrm{~m}$ sprint speed immediately post intervention. ${ }^{[1]}$ Sandell et al. checked for $30 \mathrm{~m}$ but his intervention was on sacroilaic joint and hip joint. ${ }^{[8]}$ The running speed, which was estimated according to the time it takes to cover a certain distance, was higher (mean difference of 0.062 seconds with a confidence interval

0.00 to 0.13 ) in the experimental group in the study by Sandell, Palmgren and Björndahl ${ }^{[8]}$.

In both the studies running speed improved in favour of the thrust group. A study done by Dishman et al. ${ }^{[7]}$ to check the spinal reflex excitability changes after spinal manipulation identified a transcient increase in motor evoked potential (MEP) amplitude, which lasted upto 60 seconds after spinal manipulation theory(SMT). This implies increased excitability of the corticomotor pathway after SMT and may justify the results found by Sandell ${ }^{[8]}$ and Shrier ${ }^{[1]}$ for immediate effects of thrust on running speed. Running technique has been suggested to play a key role in performance of sprints with directional changes ${ }^{[23,24]}$. Baker [26] investigated the performance differences of elite and developmental rugby league players using a similar change of direction test. It was discovered that elite players performed significantly better in tests that incorporated rapid changes of direction. To our knowledge no study has checked thrust effect on agility. We focused on straight sprint running and agility if it has any effect on athletes performance. The result that we got was our interventional group showed statistically significant improvement on agility. When compared with the control group the results were not quite significant.

Several studies did report significant immediate effects of SMT in quadriceps and ankle plantarflexion maximal voluntary isometric contraction (MVC) ${ }^{[28,29]}$, resting biceps brachii EMG ${ }^{[30]}$, and trunk joint position sense ${ }^{[31]}$ there is one low risk of bias study suggesting that a single SMT improves basketball free throw accuracy immediately post intervention compared to sham $(2.4 \%, 0.66-4.14)$, which may be relevant in high-level sport performance ${ }^{[32]}$. Only two studies provided more than one SMT over a time ${ }^{[33,34]}$, neither which had a significant effect but broad CI suggest potential issue with sample size. SMT compared to sham or other interventions does not enhance performance-based outcomes as we defined, except in a few areas. Proprioceptive afferents include Golgi tendon organs, muscle spindles, and other mechanoreceptors, such as Pacinian corpuscles and Ruffini endings. These specialized receptors are highly concentrated in axial and deep cervical muscles ${ }^{[35,36]}$. Vertebral joint dysfunctions are believed to generate central proprioceptive deficit input from those receptors, as there is impaired motion of the vertebrae. ${ }^{[37,38]}$ Spinal manipulative therapy has an influence on such dysfunctions and has been shown to improve proprioceptive processing and motor control [37, 38, 39], which could potentially influence sports performance.

Other neurophysiological findings showed decreased activity after SMT in resting paraspinal muscles on surface electromyography and in H-reflex analysis ${ }^{[7]}$. Even though we have got these results between groups, probable reason can be because of lack of sample size. On field competitive performance needs to be analysed in future. It might be interesting to analyse their performance in actual events of athletes whether it is helpful for better performance. The study done by Sandell et al. shows that decreased muscle tonic activity can be one of the plausible causes associated to the increased hip extension ${ }^{[8]}$. Once the hip flexors muscles have been identified as the main limiting structures for hip extension. However, the effects of SMT on tonic muscle activity are still controversial because data acquisition and analysis are quite different among studies. The relevance to of such changes to improvement in functional or sport performance is unclear at this time. We agree with Cerqueira et al. ${ }^{[6]}$ and Botelho et al. ${ }^{[16]}$, that studies in this field require greater methodological rigor. Specifically, studies exploring athletic performance and the effect of SMT in asymptomatic adults should focus on identifying relevant and responsive outcome measures, use adequate sample sizes, include appropriate control groups, assess outcomes over longer temporal intervals, and explore the role of athlete expectations prior to intervention. 


\section{Conclusions}

HVLA thrust with conventional training showed significant improvement within the group in elite athletes. On comparing with the control group, the results found were not significant.

\section{Clinical implications}

The question regarding the HVLA thrust to be included in athletes training still needs to be verified with more research.

\section{Future scope}

- Comparison with different treatment protocols can be considered to find the effects.

- More sample size can be included.

- Frequency dosages can be increased with further followups of athletes.

- Genderwise comparison can be done.

- Manipulation Pre, during and post competition analysis.

\section{References}

1. Shrier I, Macdonald D, Uchacz G. A pilot study on the effects of pre-event manipulation on jump height and running velocity. British Journal of Sports Medicine. 2006;40(11):947-9.

2. Keir KAI, et al. Introduction to manipulation. Physiotherapy Modalities. Br J Sp Med. 1991;25(4).

3. Coronado-Joel Bialosky-Chad Cook the temporal effects of a single session of high-velocity, lowamplitude thrust manipulation on subjects with spinal pain Physical Therapy Reviews. 2010;15(1).

4. Stump JL, Redwood D. The use and role of sport chiropractors in the National Football League: A short report. Journal of Manipulative and Physiological Therapeutics. 2002;25(3).

5. Segen's Medical Dictionary. S.v. "elite athlete." Retrieved August 172020 from https://medicaldictionary.thefreedictionary.com/elite+athlete

6. Cerqueira MS, Sales RM, Pinto CTP, Mesquita G, Moreno BGD, Filho AGDM. Effects of high-velocity low-amplitude manipulation (thrust) in athletic performance: A systematic review. Phys Ther Sport. 2016;18:e8.

7. Dishman JD, Burke J. Spinal reflex excitability changes after cervical and lumbar spinal manipulation: a comparative study. Spine J. 2003;3(3):204-12.

8. Sandell J, Palmgren PJ, Björndahl L. Effect of chiropractic treatment on hip extension ability and running velocity among young male running athletes. Journal of Chiropractic Medicine. 2008;7(2):39-47.

9. Rubinstein SM, van Middelkoop M, Assendelft WJJ, de Boer MR, van Tulder MW. Spinal manipulative therapy for chronic low-back pain (Review). The Cochrane Library 2011, 2.

10. Sheppard, Jeremy \& Young, Warren. Agility Literature Review: Classifications, Training and Testing. Journal of sports sciences. 2006;24:91932. 10.1080/02640410500457109.

11. Esposito S, Philipson S, Downie A. n.d. Spinal Adjustment Technique.

12. Miners AL, Degraauw C. A survey of Fellows in the College of Chiropractic Sports Sciences (Canada): their intervention practices and intended therapeutic outcomes when treating athletes. J Can Chiropr Assoc. 2010;54(4):282-92.

13. Rio 2016 100m men - Olympic Athletics [Internet]. International Olympic Committee. 2020 [cited 17 August 2020]. Available from:

https://www.olympic.org/rio-2016/athletics/100m-men

14. Nook D, Nook E, Nook B. Utilization of Chiropractic Care at the World Games 2013. Journal of Manipulative and Physiological Therapeutics. 2016;39(9):693-704.

15. Miners AL. Chiropractic treatment and the enhancement of sport performance: a narrative literature review. J Can Chiropr Assoc. 2010;54(4):210-21.

16. Botelho M, Alvarenga B, Molina N, Ribas M, Baptista A. Spinal Manipulative Therapy and Sports Performance Enhancement: A Systematic Review. Journal of Manipulative and Physiological Therapeutics. 2017;40(7):535-543.

17. Pollard H, Ward G. Strength change of quadriceps femoris following a single manipulation of the L3/4 vertebral motion segment: a preliminary investigation. $\mathbf{J}$ Neuromusculoskel Syst. 1996;4(4):137-44.

18. Méndez-Sánchez R, González-Iglesias J, SánchezSánchez J, PuenteGonzález A. Immediate Effects of Bilateral Sacroiliac Joint Manipulation on Plantar Pressure Distribution in Asymptomatic Participants. The Journal of Alternative and Complementary Medicine. 2014;20(4):251-257.

19. Deutschmann KC, Jones AD, Korporaal CM. A nonrandomised experimental feasibility study into the immediate effect of three different spinal manipulative protocols on kicking speed performance in soccer players. Chiropractic \& Manual Therapies. 2015;(23:1).

20. Gabbett Tim, Sheppard Jeremy, Polglaze T. Speed and Agility, 2013.

21. Per Gunnar Brolinson, et al. Precompetition Manipulative Treatment and Performance Among Virginia Tech Athletes During 2 Consecutive Football Seasons: A Preliminary, Retrospective Report JAOA, 2012;112:9.

22. Semenick D. The T-test. NSCA J. 1990;12(1):36-37.

23. Sheppard JM, Dawes JJ, Jeffreys I, Spiteri T, Nimphius S. Broadening the view of agility: A scientific review of the literature.

24. Bompa T. Theory and methodology of training. Dubuque, Iowa: Kendall-Hunt, 1983.

25. Sayers M. Running techniques for field sport players, in: Sports Coach. 2000, pp 26-27.

26. Baker D. A comparison of running speed and quickness between elite professional and young rugby league players. Strength and Conditioning Coach. 1999;7:3-7.

27. Pasquier M, Daneau C, Marchand A, Lardon A, Descarreaux M. Spinal manipulation frequency and dosage effects on clinical and physiological outcomes: a scoping review. Chiropractic \& Manual Therapies. 2019;27(1).

28. Christiansen TL, Niazi IK, Holt K, Nedergaard RW, Duehr J, Allen K, et al. The effects of a single session of spinal manipulation on strength and cortical drive in athletes. Eur J Appl Physiol. 2018;118(4):737-49.

29. Grindstaff TL, Hertel J, Beazell JR, Magrum EM, Ingersoll CD. Effects of lumbopelvic joint manipulation on quadriceps activation and strength in healthy individuals. Man Ther. 2009;14(4):415-20. 
30. Dunning J, Rushton A. The effects of cervical highvelocity low-amplitude thrust manipulation on resting electromyographic activity of the biceps brachii muscle. Man Ther. 2009;14(5):508-13.

31. Learman KE, Myers JB, Lephart SM, Sell TC, Kerns GJ, Cook CE. Effects of spinal manipulation on trunk proprioception in subjects with chronic low Back pain during symptom remission. J Manip Physiol Ther. 2009;32(2):118-26.

32. Costa SMV, Chibana YET, Giavarotti L, Compagnoni DS, Shiono AH, Satie J, et al. Effect of spinal manipulative therapy with stretching compared with stretching alone on full-swing performance of golf players: a randomized pilot trial. J Chiropr Med. 2009;8(4):165-70.

33. Gavin D. The effect of joint manipulation techniques on active range of motion in the mid-thoracic spine of asymptomatic sybjects. J Man Manip Ther. 1999;7(3):114-22.

34. Olson E, Bodziony M, Ward J, Coats J, Koby B, Goehry D. Effect of lumbar spine manipulation on asymptomatic cyclist sprint performance and hip flexibility. J Chiropr Med. 2014;13(4):230-8.

35. Kulkarni V, Chandy MJ, Babu KS. Quantitative study of muscle spindles in suboccipital muscles of human foetuses. Neurol India. 2001;49(4):355-359.

36. Boyd-Clark LC, Briggs CA, Galea MP. Muscle spindle distribution, morphology, and density in longus colli and mutifidus muscle of the cervical spine. Spine (Phila Pa 1976. 2002;27(7):694-701.

37. Haavik-Taylor H, Murphy B. Cervical spine manipulation alters sensorimotor integration: a somatosensory evoked potential study. Clin Neurophysiol. 2007;118(2):391-402.

38. Haavik H, Murphy B. The role of spinal manipulation in addressing disordered sensorimotor integration and altered motor control. J Electromyogr Kinesiol. 2012;22(5): 768-776.

39. Haavik Taylor H, Murphy B. The effects of spinal manipulation on central integration of dual somatosensory input observed after motor training: a crossover study. J Manipulative Physiol Ther. 2010;33(4):261-272.

40. Brenner AK, Gill NW, Buscema CJ, Kiesel K. Improved Activation of Lumbar Multifidus Following Spinal Manipulation: A Case Report Applying Rehabilitative Ultrasound Imaging. Journal of Orthopaedic \& Sports Physical Therapy. 2007;37(10):613-9.

41. Brolinson PG. Precompetition Manipulation: Placebo or Performance Enhancer? Clinical Journal of Sport Medicine. 2003;13(2):69-70.

42. Per Gunnar Brolinson, et al. Precompetition Manipulative Treatment and Performance Among Virginia Tech Athletes During 2 Consecutive Football Seasons: A Preliminary, Retrospective Report JAOA. 2012;112:9.

43. Hedlund S, Nilsson H, Lenz M, Sundberg T. Effect of Chiropractic Manipulation on Vertical Jump Height in Young Female Athletes with Talocrural Joint Dysfunction: A Single-Blind Randomized Clinical Pilot Trial. Journal of Manipulative and Physiological Therapeutics. 2014;37(2):116.
44. Perle SM. Mechanical force spinal manipulation increases trunk muscle strength assessed by electromyography: A comparative clinical trial. Journal of Manipulative and Physiological Therapeutics. 2002;25(5):345.

45. Haldeman S. Point Of View: Electromyographic Responses of Back and Limb Muscles Associated With Spinal Manipulative Therapy. Spine. 1999;24(2):153.

46. Ditcharles S, Yiou E, Delafontaine A, Hamaoui A. Short-Term Effects of Thoracic Spine Manipulation on the Biomechanical Organisation of Gait Initiation: A Randomized Pilot Study. Frontiers in Human Neuroscience, 2017, 11. 